\title{
Shielding considerations for ESS LoKI
}

\author{
Gábor Náfrádi, Anton Orszulik, Jacob Cooper, Ben Hicks, \\ David Turner, Will Halcrow, Andrew Jackson and Steven Lilley
}

\section{Published version information}

Citation: G Náfrádi et al. 'Shielding considerations for ESS LoKI.' Journal of Neutron Research, vol. 22, no. 2-3 (2020): 119-129. Is in proceedings of: 23rd Meeting of the International Collaboration on Advanced Neutron Sources (ICANS-XXIII), Chattanooga, Tennessee, United States, 13-18 Oct 2019.

DOI: $\underline{10.3233 / J N R-200152}$

The final publication is available at IOS Press through DOI above.

This version is made available in accordance with publisher policies. Please cite only the published version using the reference above. This is the citation assigned by the publisher at the time of issuing the AAM. Please check the publisher's website for any updates. 


\title{
1 Shielding Considerations for ESS LoKI
}

\author{
2 Gábor Náfrádia, ${ }^{\mathrm{a}}$, Anton Orszulik ${ }^{\mathrm{a}}$, Jacob Cooper ${ }^{\mathrm{a}}$, Ben Hicks ${ }^{\mathrm{a}}$, David Turner ${ }^{\mathrm{a}}$, Will Halcrow ${ }^{\mathrm{a}}$, \\ 3 Andrew Jackson ${ }^{\mathrm{b}}$, and Steven Lilley ${ }^{\mathrm{a}}$, \\ 4 a ISIS Facility, Rutherford Appleton Laboratory, Chilton, Didcot, Oxfordshire OX11 0QX, United \\ 5 Kingdom \\ $6 \quad$ E-mail: gabor.nafradi@stfc.ac.uk,william.halcrow@stfc.ac.uk, steven.lilley@stfc.ac.uk \\ 7 buropean Spallation Source, SE-211 00 Lund, Sweden \\ 8 E-mails: andrew.jackson@esss.se
}

Abstract. LoKI is a SANS instrument being built by STFC ISIS as an in-kind contribution to ESS. According to the recent schedule, LoKI, is one of the first three instruments of ESS that is going to contribute to early science from 2023. MCNP calculations were carried out to determine the required shielding around different sections of the instrument. For the geometry development, SuperMC/MCAM was used. Highlights from the neutronics calculations based on the most frequent classes of possible events are presented here. These include heavy shutter performance, collimator section shielding and detector tank shielding. Activation and background calculations are not detailed in this paper.

Keywords. ESS, LoKI, radiation shielding, MCNP

\section{Introduction}

The European Spallation Source (ESS) [1] is being built by thirteen European countries in Lund, Sweden. The participating countries are contributing to the project as in-kind partners. One of the inkind contribution of the United Kingdom (UK) is LoKI [2], which is a Small Angle Neutron Scattering (SANS) instrument. LoKI is one of the first three instruments of ESS therefore, according to the plans it will contribute to the early science stage of ESS from 2023.

ESS is going to be a massive facility with extremely high radiation levels therefore shielding is a crucial point. The shielding calculations presented here followed the ESS guidelines [3]. These guidelines set the radiation dose limits for different radiologically categorized areas [4]. For the dose rate limits a safety factor of 2 should be applied if the neutronics calculations are carried out with a Monte Carlo method based code. For every instrument a list of typical operational and incident scenarios should be identified and categorized based on their frequency of appearance. The shielding design should consider the first two most frequent group of scenarios. The so called H1 group contains the normal operation scenarios while the group called $\mathrm{H} 2$ contains the scenarios that can occur at least once per hundred years but are not considered as H1 [5]. During H1 events the maximal accepted combined neutron and gamma dose rate limit in supervised areas like areas around LoKI is $3 \mu \mathrm{Sv} / \mathrm{h}$. A Monte Carlo based shielding design therefore should aim to have a $1.5 \mu \mathrm{Sv} / \mathrm{h}$ dose rate limit. For $\mathrm{H} 2$ events, the limit is 20 $\mathrm{mSv} / \mathrm{event}$. The less frequent scenarios are not considered in the shielding design of the instruments.

LoKI is a so called short instrument located at the N7 port of ESS. The total length of LoKI is about $37 \mathrm{~m}$ while a typical length of a long instrument is about $150 \mathrm{~m}$. The neighboring instruments will be the Test Beamline and FREIA [6] which is also a UK developed instrument. LoKI starts with the first

*Corresponding author. E-mail: gabor.nafradi@stfc.ac.uk 
guide section from the inner surface of the monolith at a distance of $2 \mathrm{~m}$ from the target. The guide in the monolith is bended downwards to reduce high energy neutron contribution in the beam (see Figure 1). After the monolith the guide is straight, the next bended section is located in the bunker wall where the guide bends upwards. This second bended section secures the two-times out of line of sight requirement and separating the high energy neutrons traveling down the beam from the low energy confined beam. The guides are straight after the bunker.

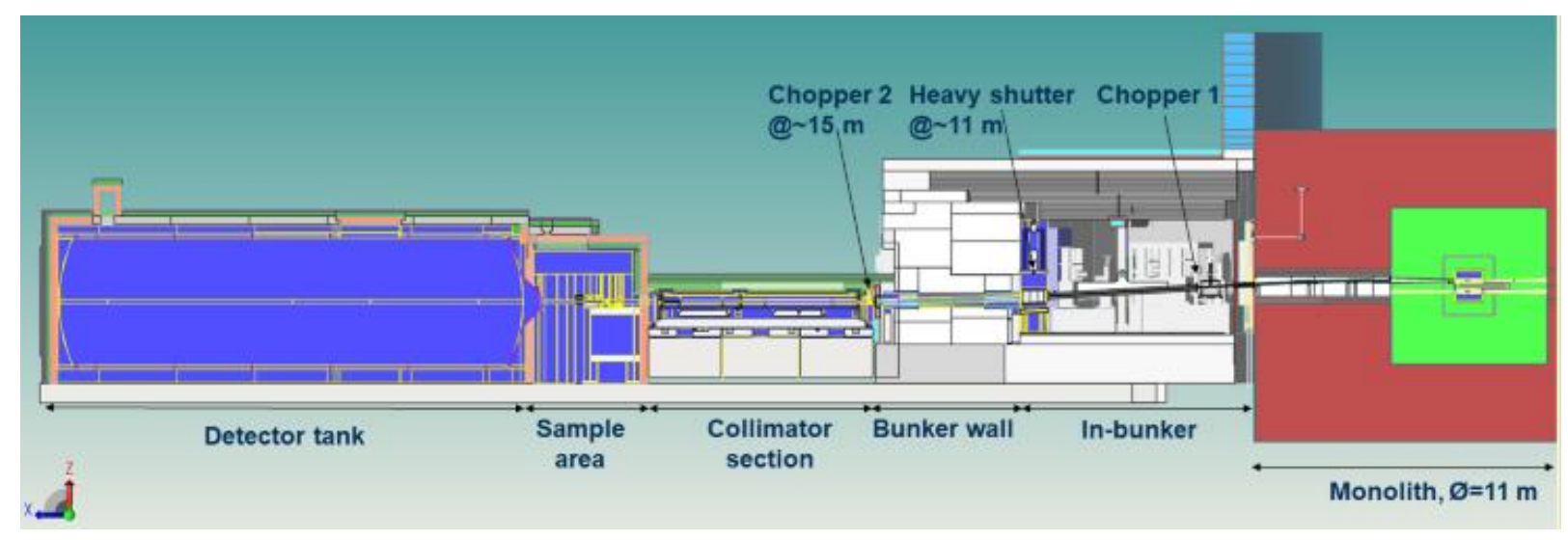

Figure 1 Layout of LoKI along the beam, cross sectional view

The neutronics calculations were carried out with Monte Carlo N-Particle code (MCNP) [7]. The MCNP model was made from CAD models using the CAD-to-MCNP converter module of SuperMC $[8,9]$. The released and distributed version of MCNP does not contain the physics of the neutron guides therefore MCNP was used together with McSTAS [10,11,12,13] if the low energy confined beam parameters were needed. The confined beam can interact with slits, chopper blades, samples etc. determined by the H1-H2 scenarios. In these cases, first a McSTAS calculation was used and the neutron parameters were determined just in front of the obstacles. In a later MCNP calculation, the low energy neutrons were started with adjusted parameters according to the McSTAS calculations.

A neutron source term on the inner surface of the monolith was provided by ESS containing neutron energies up to $500 \mathrm{MeV}$ [14]. This source term was used for the high energy transport calculations carried out with MCNP mostly for the heavy shutter design.

The shielding design is prioritized by ESS. The first priority is keeping the dose rate limitations in the differently classified areas while the activation of the components and the instrument background are the second and third priorities respectively. Here the problems related to activation or the instrument background are not detailed.

The shielding design is an ongoing process. The work presented here covers the design of the instrument heavy shutter but not the bunker wall and the roof of the bunker since these are ESS responsibilities. The work also covers the shielding considerations for the instrument sections located outside of the bunker namely: the second chopper and collimator section, sample area and detector tank area. However, due to length restrictions, only highlights will be shown, the work in more details will be available in the documentation of the instrument design processes. 
This section contains those scenarios that were used for the calculations [5]. The scenarios are collected into four groups.

Heavy shutter related scenarios:

- Heavy shutter is shut, the high energy neutron beam interacts with the shutter (H1-1)

- Heavy shutter is shut, the low energy (confined) beam interacts with the shutter (H1-1)

- Heavy shutter is open, the high energy beam interacts with the bunker feedthrough. (In the open state the low energy beam was not considered in MCNP as it wouldn't be transported correctly) (H1-1)

Collimator and $2^{\text {nd }}$ chopper area:

- Chopper\#2 blades are hit by the neutron beam (H1-3)

- Slits are closed and are made of B-10 or Cd (H1-4)

- Continuous guide loss of neutrons on the guide surface (H1-6)

- Gate valve (aluminum) is closed (H2-6)

Sample area:

- $\mathrm{H}_{2} \mathrm{O}$ as good neutron scatterer in the sample position (H1-8, $\left.\mathrm{H} 2-2\right)$

- $\mathrm{Cd}$ as good neutron-gamma converter in the sample position (H1-12)

- Gd as good neutron-gamma converter in the sample position (H1-7)

- $\mathrm{Al}$ as structural component in the sample position (H1-10, H2-5)

- Steel as structural component in the sample position (H1-10)

- Gate valve is closed (aluminum) (H2-6)

- Fast shutter is closed $\left(\mathrm{B}_{4} \mathrm{C}\right)(\mathrm{H} 1-9)$

Detector tank area:

- $\mathrm{B}_{4} \mathrm{C}$ or $\mathrm{Cd}$ absorbers in various positions along the beam in the detector vessel (H1-15)

- $\mathrm{Cd}$ as good neutron-gamma converter in the sample position (H1-12)

- $\mathrm{Gd}$ as good neutron-gamma converter in the sample position (H1-7)

- $\mathrm{H}_{2} \mathrm{O}$ as good neutron scatterer in the sample position (H1-8)

- $\mathrm{H}_{2} \mathrm{O}$ as a good neutron scatterer in the sample position and a circular $\mathrm{Cd}$ film representing a detector mask is in the detector tank (H1-13)

\section{Heavy shutter calculations}

The heavy shutter has two states, an open and a closed one. In closed state the shutter should block the beam, securing that the vast majority of the radiation is attenuated properly and the dose rate levels can be smaller than the limits outside of the bunker wall. To check this, two types of calculations were carried out. A high energy beam transport calculation when the neutrons are started from the inner surface of the monolith and a low energy beam calculation when the small energy neutrons were shot into the closed shutter. The high energy beam calculation was repeated with an open shutter as well to check the significance of the high energy neutron dose rate outside of the bunker. The gamma and neutron dose rate maps were converted with the standard ESS flux to dose rate conversion factors [3]. 
The heavy shutter is a laminated structure, the cross section of it can be seen in Figure 2. The first $\mathrm{B}_{4} \mathrm{C}$ layer intends to absorb the low energy beam. Slowing of the high energy neutrons occurs in the next three layers. The purpose of the last $\mathrm{B}_{4} \mathrm{C}$ layer is to remove the slowed neutrons passing through the laminated structure. The borated polyethylene at the rear and the aluminum layer in front surround the steel middle block and act as a gamma shielding in the case of activation of steel. That can be advantageous during maintenance procedures.

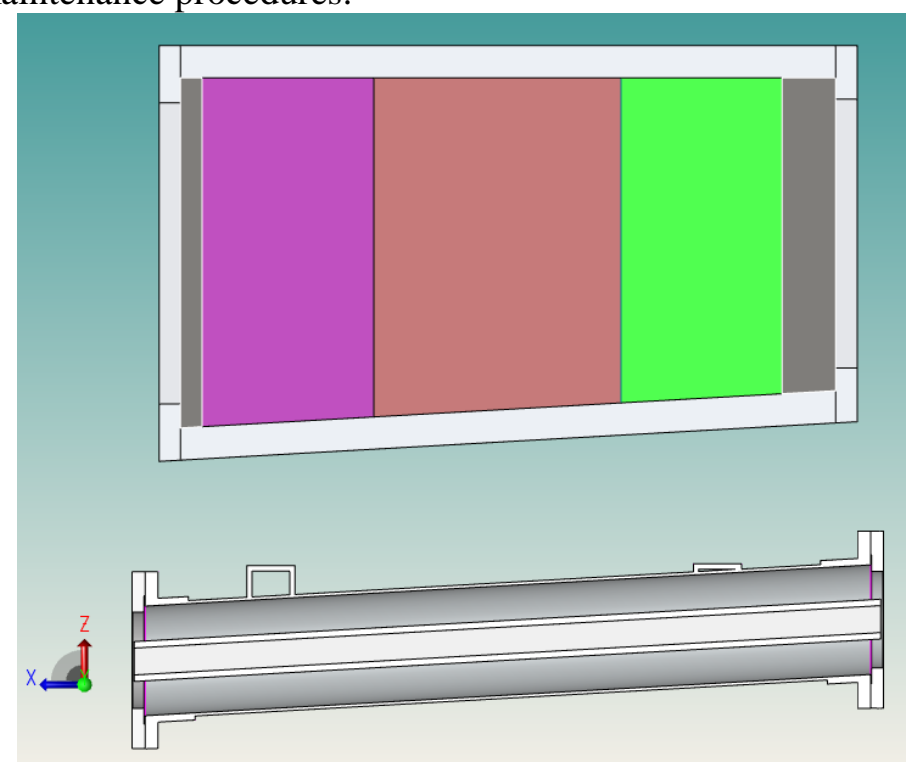

Figure 2 The cross section of the heavy shutter and the guide section underneath. Neutrons are propagating from right to left. Black layers at the front face $(5 \mathrm{~cm})$ and at the rear $(2 \mathrm{~cm})$ of the shutter are made of $\mathrm{B}_{4} \mathrm{C}$. The green layer $(15 \mathrm{~cm})$ is made of aluminum. The brown layer in the middle $(23 \mathrm{~cm})$ is made of structural steel while the pink layer $(16 \mathrm{~cm})$ is made of borated polyethylene. The grey frame is made of steel around the laminated structure.

\subsection{High energy beam calculation}

The procedure for calculating the high energy beam transport was the following. First in a reduced density model a mesh based neutron weight window map was generated with the MCNP-s inbuilt weight window generator, where the aimed F5 tally was outside of the bunker, lying on the beam axis. In a second normal density calculation that weight window was used for shepherding the particles weight towards the tally mentioned above. Weight window technique was also combined with exponential transform through the closed shutter cells.

In the calculations only neutron source terms were used. The first guide section in the monolith already bended and from the shutter there is no direct line of sight for the gamma photons to the moderator.

The neutron source at the monolith was a multidirectional source (see Figure 3). 


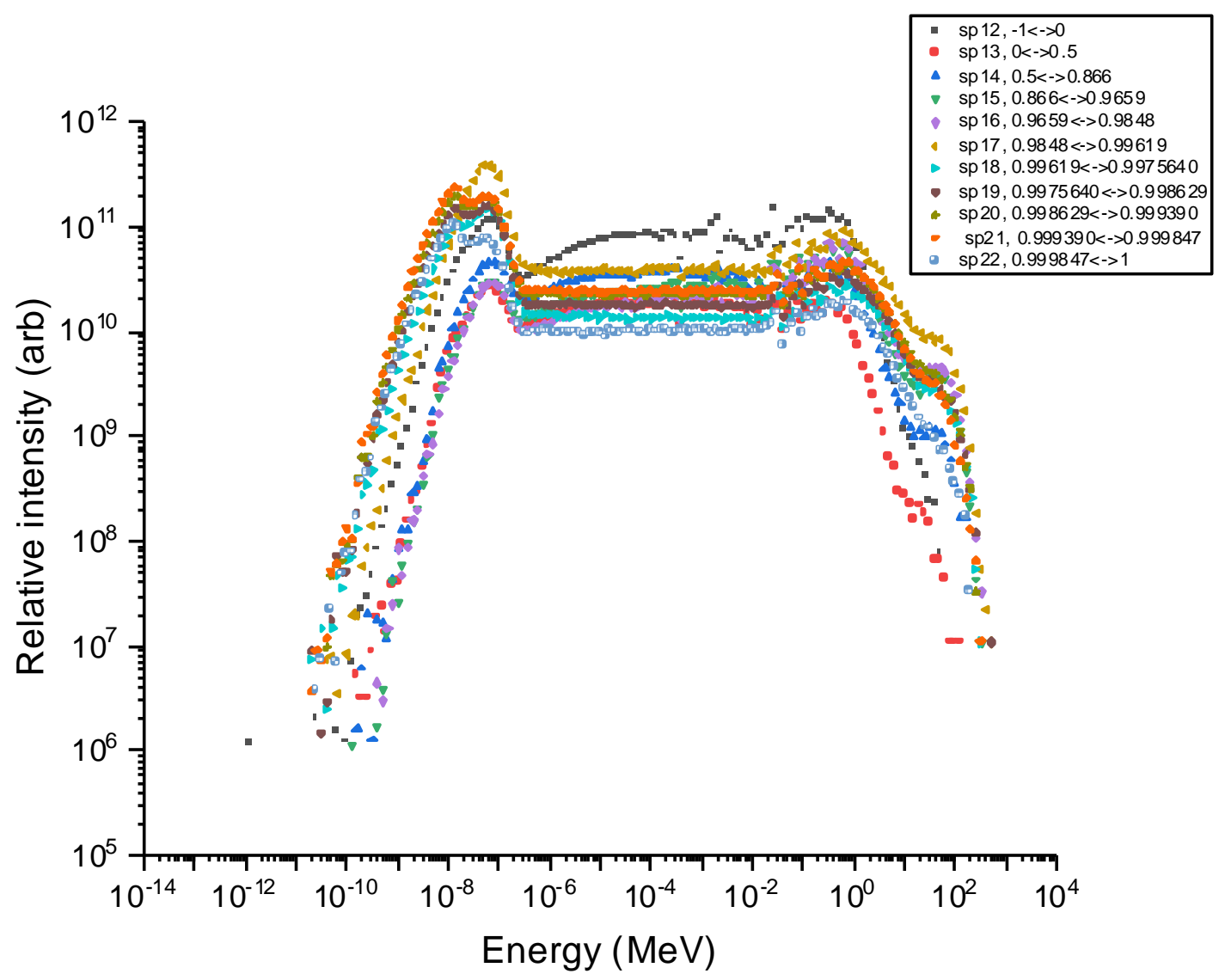

Figure 3 The relative source intensities in different directions as a function of neutron energy

The source intensity in different cosine intervals denoted with different "sp" cards as a function of energy. Here 1 means forward direction (towards the shutter). The maximum energy for the started neutrons in this source term is about $501 \mathrm{MeV}$. In the calculation: neutrons, photons, positive pions, protons, deuterons, tritons, helions and alpha particles were transported. For photons the photo-nuclear reactions were enabled on the phys card and the maximal photon energy was limited to $100 \mathrm{MeV}$. For other particles the maximal energy limited to $510 \mathrm{MeV}$. The default high energy physics cards (lca and lea) were set, applying Bertini and ISABEL models with the use of pre-equilibrium model after intranuclear cascade.

12 The neutrons were started from an $8 \mathrm{~cm}$ x $10 \mathrm{~cm}$ surface centered around the LoKI beam center on the 13 inner surface of the Monolith. The size of the source is larger than the beam size $(2.5 \mathrm{~cm} \times 3 \mathrm{~cm})$ which 14 ensures a conservative estimation taking into account the leaking paths around the guide in the Neutron 15 Beam Extraction System (NBEX). The neutrons transported by MCNP. The obtained neutron dose rate 16 maps can be seen in Figure 4. The neutron beam nicely attenuated by the shutter, the neutron dose rate 17 drops far below the $1.5 \mu \mathrm{Sv} / \mathrm{h}$, which is the limit on the other side of the bunker wall. 

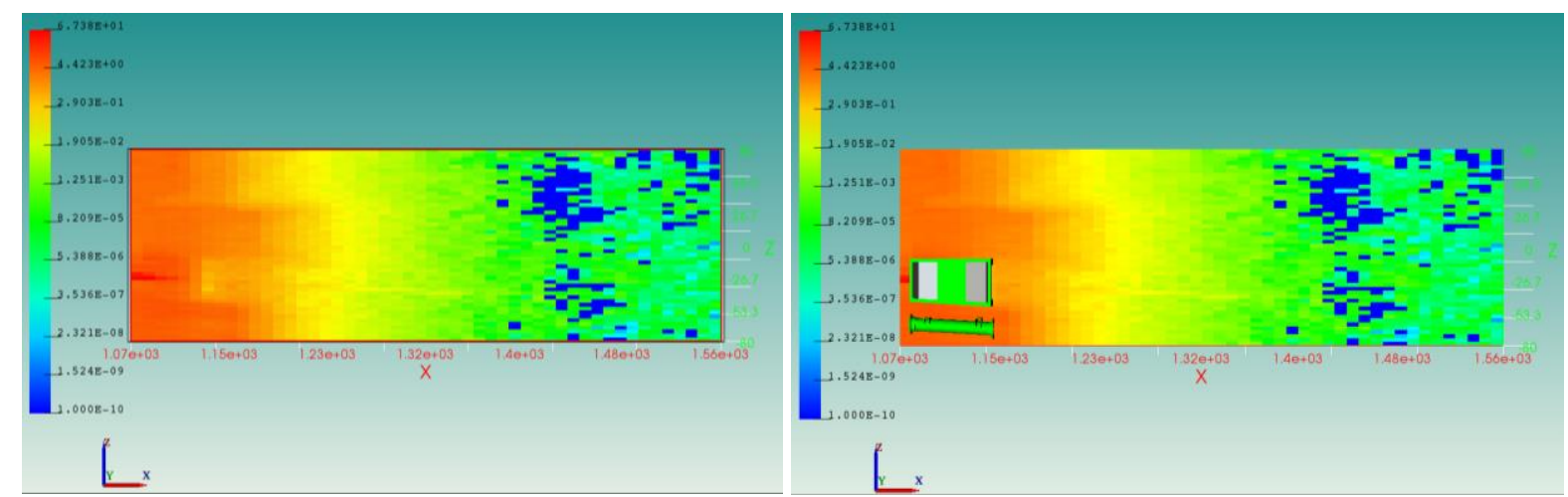

Figure 4 Cross section of the neutron dose rate map along the beam when the heavy shutter is in a closed state. The colorbar is in $\mu$ Sv/h. On the right the heavy shutter and the guide section beneath are also shown for better orientation on the figure. Neutrons are propagating from left to right. All axes dimension is $\mathrm{cm}$. The values of the colorbar are in $\mu \mathrm{Sv} / \mathrm{h}$.

\subsubsection{Open shutter calculations}

The high energy calculation was repeated with the heavy shutter open to calculate the beam attenuation in the bunker wall. The obtained neutron dose rate map can be seen in Figure 5. The neutron dose rate from the high energy beam is far below $1.5 \mu \mathrm{Sv} / \mathrm{h}$ on the other side of the bunker (from about $\mathrm{x}=1.5 \mathrm{E} 03 \mathrm{~cm}$ ) wall. This indicates that the contribution of high energy neutron beam on the outer side of the bunker is negligible compared to the confined beam related dose rates.

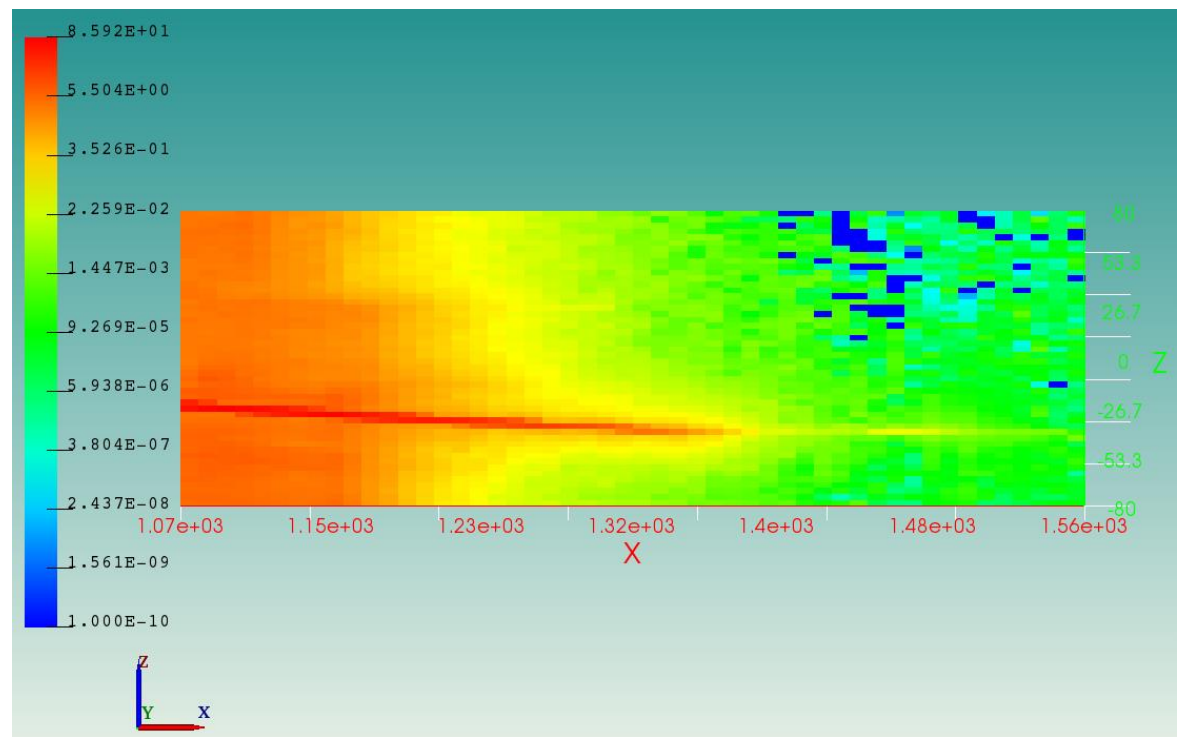

Figure 5 Cross section of the neutron dose rate map along the beam when the shutter is open. All axes dimension is $\mathrm{cm}$. The values of the color bar are in $\mu \mathrm{Sv} / \mathrm{h}$. 
2 For the low energy confined beam a different neutron source term was used. This source term was a 3 monodirectional $2.5 \mathrm{~cm} \times 3 \mathrm{~cm}$ rectangular surface source, pointing along the beam axis towards the 4 shutter. The relative intensity of the source as a function of neutron energy can be seen in Figure 6.

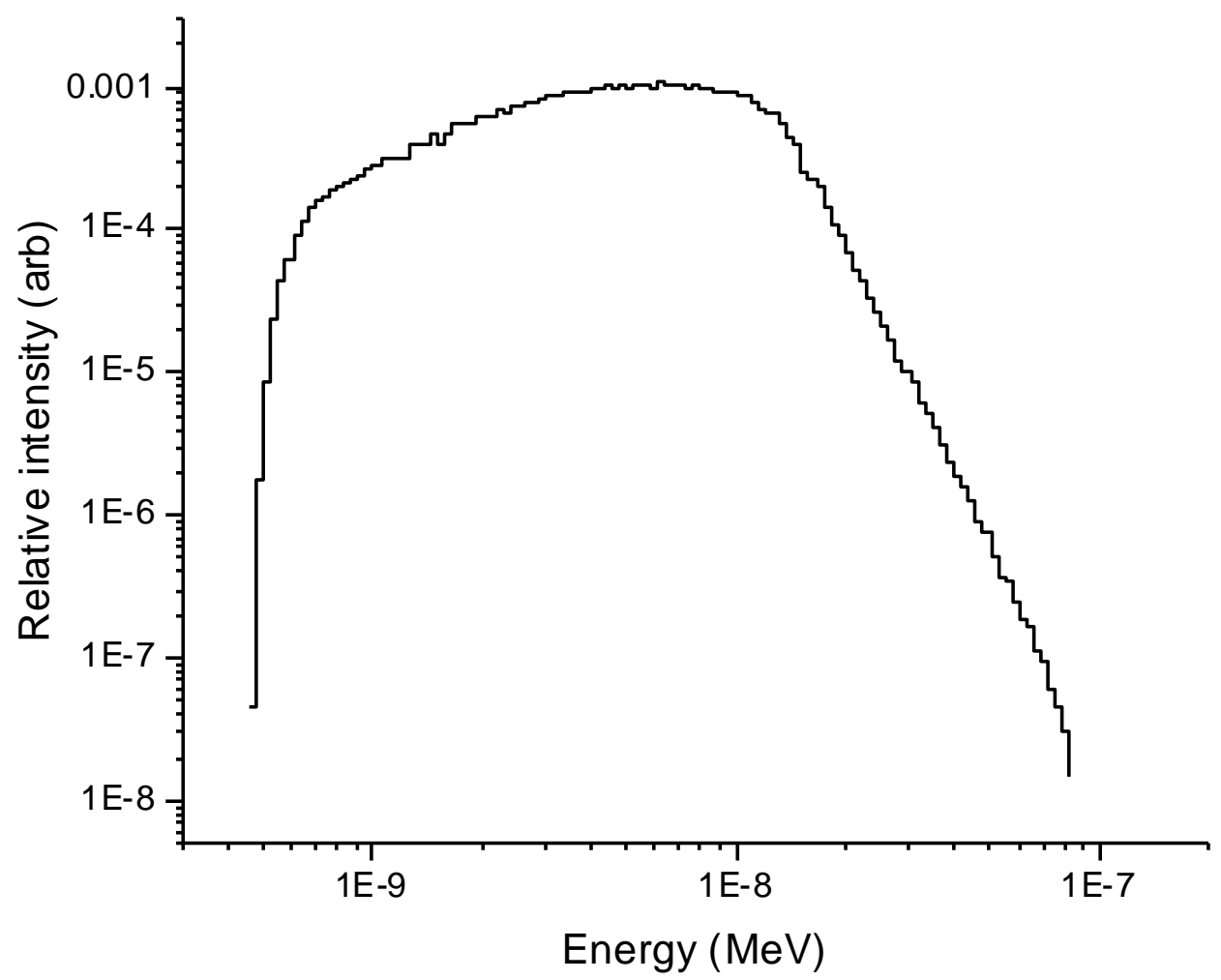

The low energy neutron calculation was analogue. The same particles were followed as in the were used from ENDF/B-VII.1. If for example photon production data was missing than other older tables were used.

The low energy neutron beam was shot into the closed heavy shutter. The neutron dose rate map and 14 the gamma dose rate map is shown in Figure 7 and Figure 8 

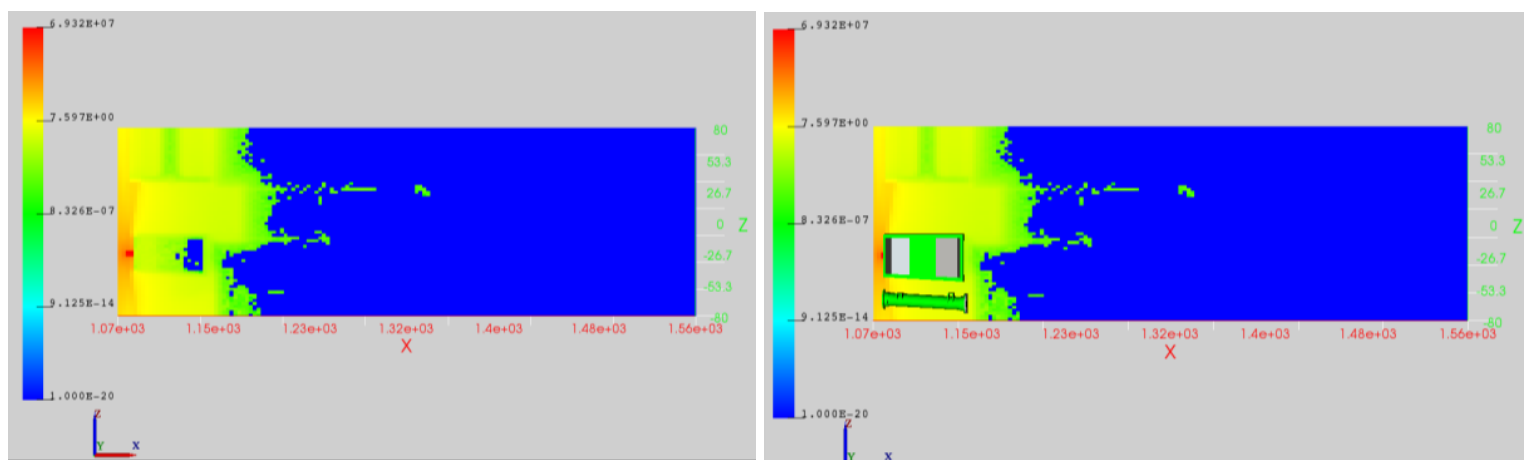

Figure 7 Cross section of the neutron dose rate map of the closed heavy shutter when the confined low energy neutron beam hits it. On the right with the heavy shutter, to help orientation. All axes dimension is $\mathrm{cm}$. The values of the color bar are in $\mu \mathrm{Sv} / \mathrm{h}$.
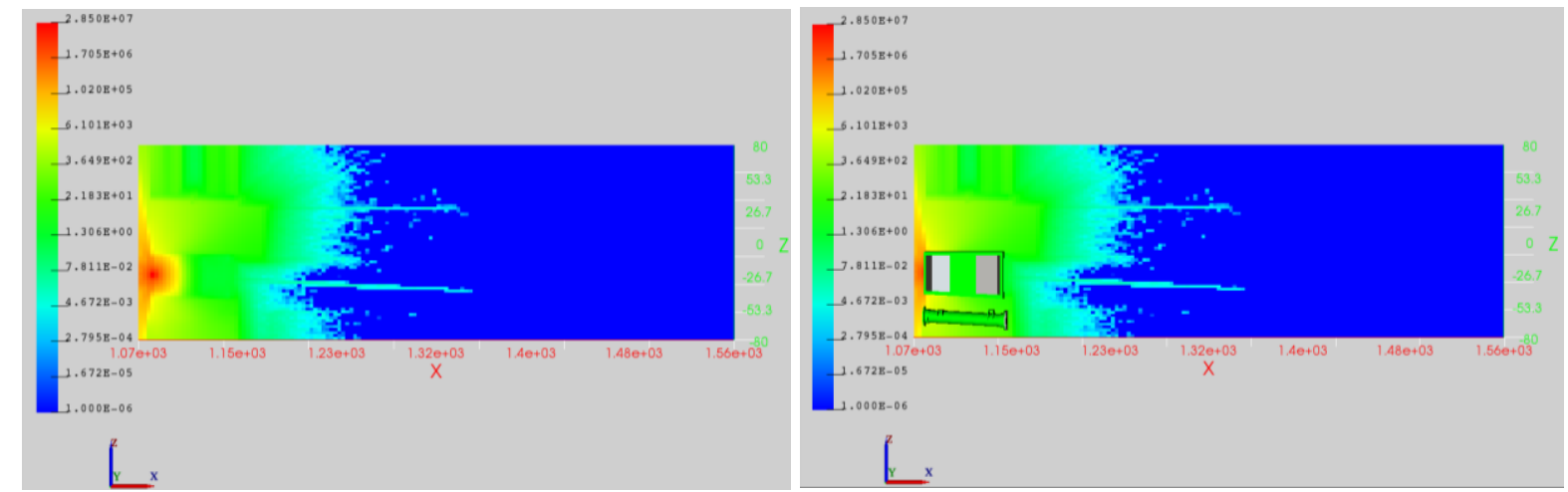

Figure 8 Cross section of the gamma dose rate map of the closed heavy shutter when the confined low energy neutron beam hits it. On the right the same plot with the heavy shutter, to help orientation. All axes dimension is $\mathrm{cm}$. The values of the color bar are in $\mu \mathrm{Sv} / \mathrm{h}$.

The neutrons were stopped at the $\mathrm{B}_{4} \mathrm{C}$ layer as expected, only a relatively small portion of scattered field is generated around the shutter. The gamma radiation penetrates more into the bunker wall but it dies out far away from the opposite side of the bunker wall. Both the gamma and neutron dose rates are orders of magnitude higher than that of the high energy neutron beam.

\section{Collimator section calculations}

As it can be seen in section 3.2.1 the dose rate from high energy beam is attenuated well through the bunker wall. In the collimator section different $\mathrm{H} 1-\mathrm{H} 2$ scenarios were taken into account but in every case the confined beam is considered as a source term, only the obstacles are varying in the beam.

As an initial step the collimator section was surrounded with a thick bulk shielding. To estimate the required shielding, neutron and gamma dose rate maps were calculated. From these dose rate maps the contour surface of the $1.5 \mu \mathrm{Sv} / \mathrm{h}$ dose rate was obtained. The aim is to find the minimal required shielding that contains the contour surface. As an example, the H1-6 scenario was considered in section 4.1. 
The neutron loss was modelled with two different methods. First a neutron line source was implemented into the axis of the beam and the neutrons were started isotropically with an assumed $1 \% / \mathrm{m}$ loss intensity. This source approximates the neutron conversion to gamma radiation in the aluminum substrate of the guide. The second method is a gamma line source in the axis of the beam emitting nickel or titanium prompt gamma lines. This source term approximates the neutron to gamma conversion in the multilayer of the guide. The neutron loss intensity for such a source was determined by Rodion Kolevatov with McSTAS [15]. For our simulation the $15 \mathrm{~m}-20 \mathrm{~m}$ interval is interesting, see 9 Figure 9.

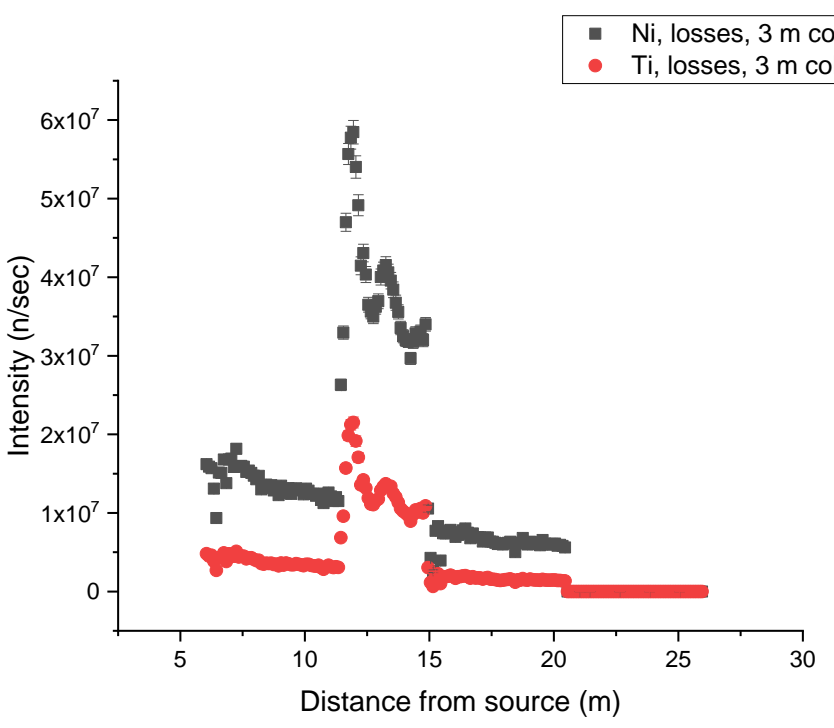

Figure 9 Neutron losses in the coating material of the guide as a function of distance.

The results with the neutron line source can be seen on Figure 10 and Figure 11. In Figure 10 one can see a yellow contour surface belonging to the $1.5 \mu \mathrm{Sv} / \mathrm{h}$ dose rate. The colourful planes (see the colour code) are showing the relative error around the contour. The relative errors are small close to the contour surface therefore these contours can be used to determine the minimal required shielding thicknesses. If one plots different shielding blocks over the contours (see Figure 11) the minimal thickness of the shielding that incorporates the contour can be found. The contours are closed which means they can be surrounded with shielding. The estimated thickness for structural steel shielding is about $30 \mathrm{~cm}$. This thickness however can vary if the distance is modified between the source and the inner surface of the shielding. 
1
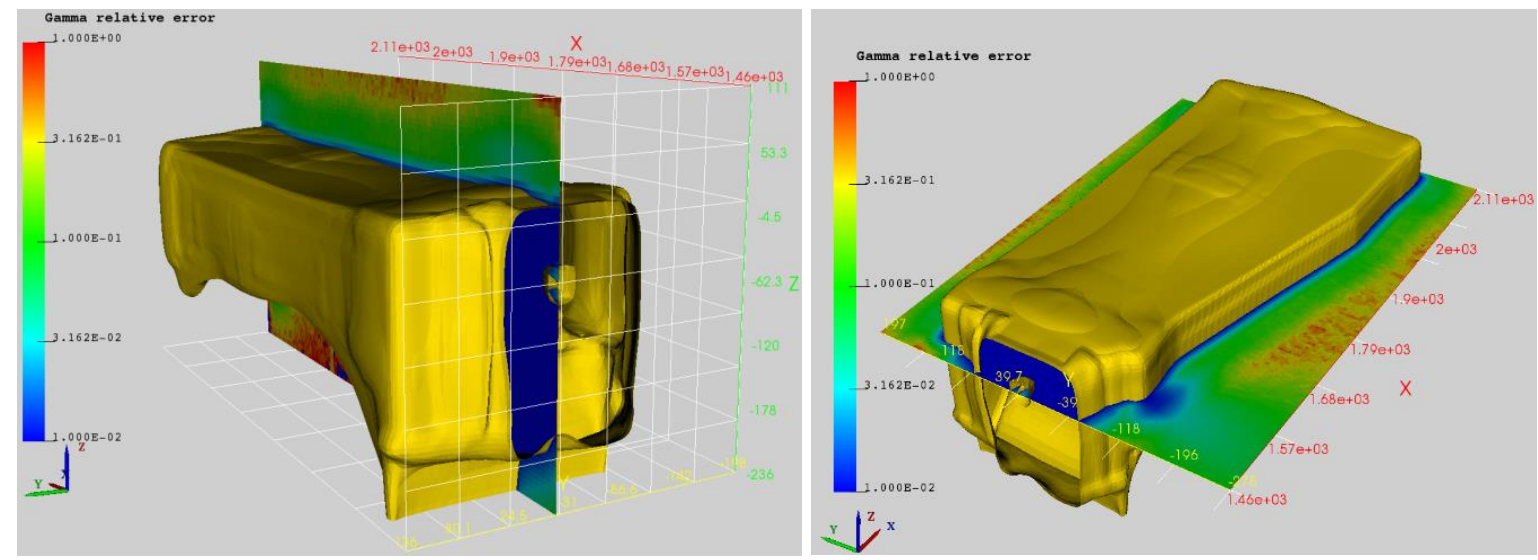

Figure 10 Gamma dose rate $1.5 \mu \mathrm{Sv} / \mathrm{h}$ contour plots around the collimator section together with the cross section of the relative error plots. All axes dimension is $\mathrm{cm}$.
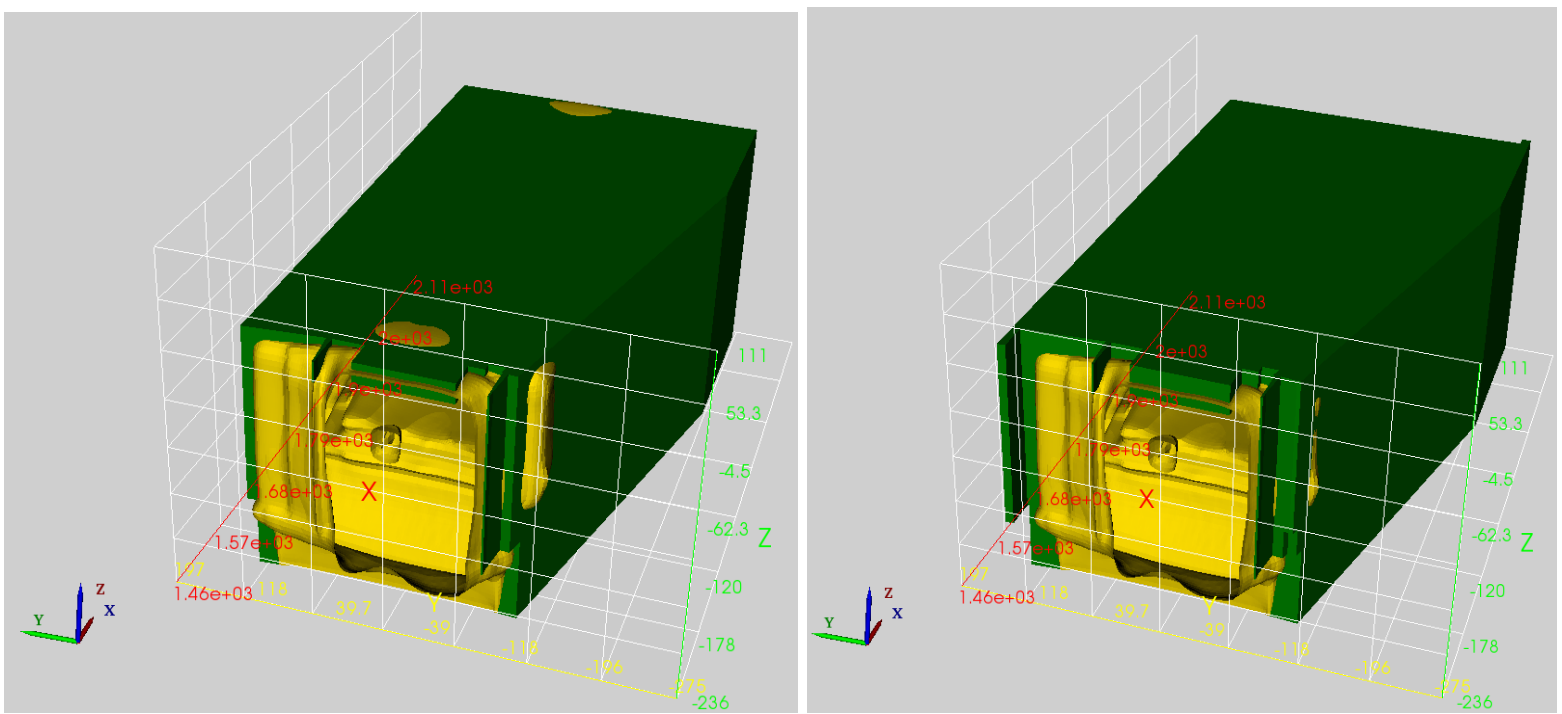

Figure 11 Left, $20 \mathrm{~cm}$ of steel shielding. Right, $30 \mathrm{~cm}$ of steel shielding. All axes dimension is $\mathrm{cm}$

Similarly, the results of the more intense Ni coating gamma radiation can be seen in Figure 12 Applying $20 \mathrm{~cm}$ of steel shielding seems satisfying except two hot spots, one on the top FREIA side and one above the $2^{\text {nd }}$ Chopper, see Figure 13. 


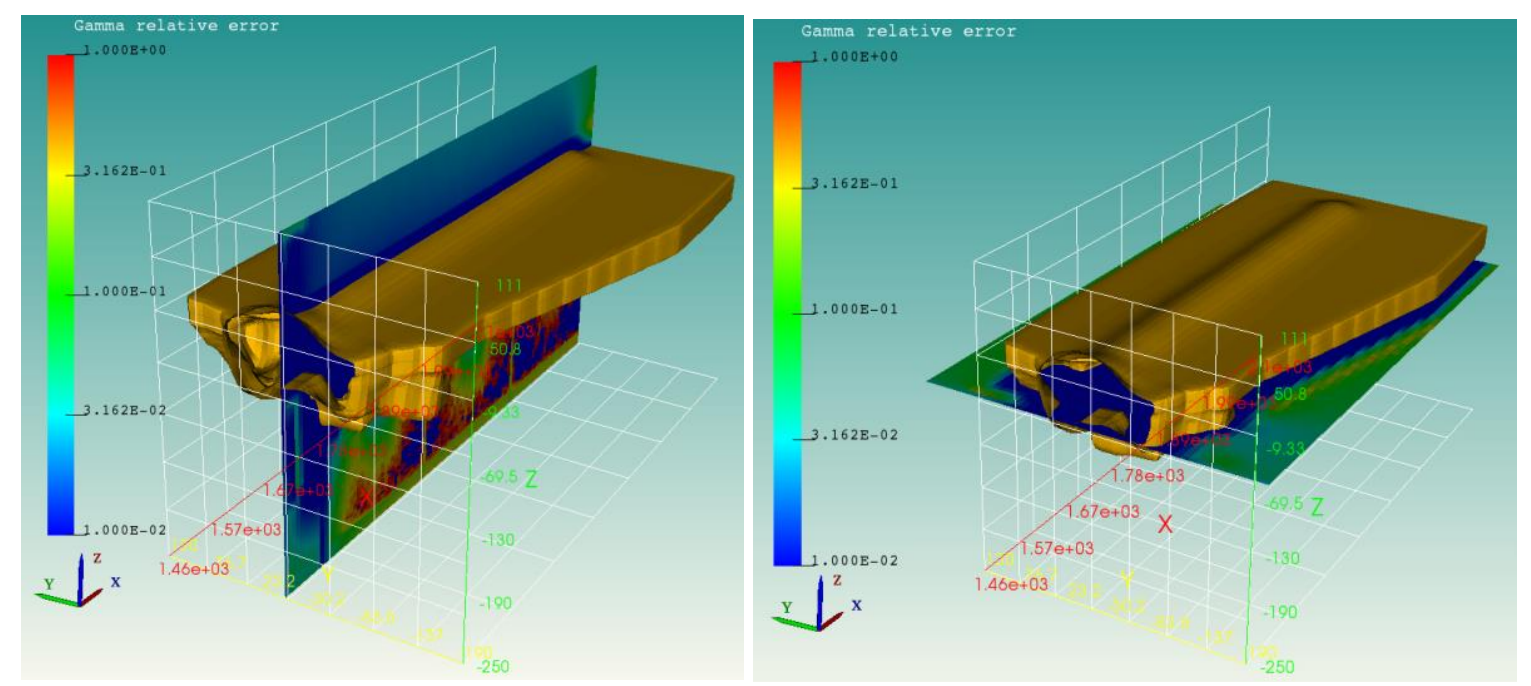

1

Figure $121.5 \mu \mathrm{Sv} / \mathrm{h}$ gamma dose rate contours with the cross sectional view of the relative error map along the beam. All axes dimension is $\mathrm{cm}$.

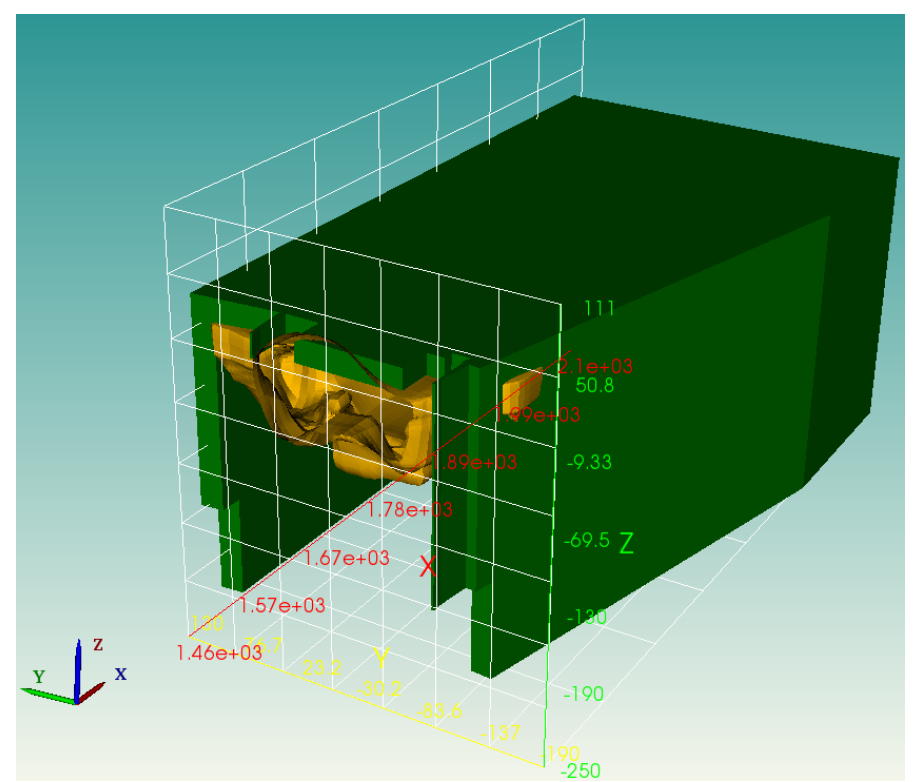

Figure $131.5 \mu \mathrm{Sv} / \mathrm{h}$ gamma dose rate contour from the Ni coating with $20 \mathrm{~cm}$ of steel shielding. All axes dimension is $\mathrm{cm}$.

\section{Sample area and detector tank area calculations}

The radiation analysis of these two sections are coupled since there is no wall separating the two sections. As an example, consider the H1-7 scenario when a Gd sample is placed into the sample position. The contour surface of the $1.5 \mu \mathrm{Sv} / \mathrm{h}$ gamma dose rate can be seen in Figure 14. The obtained contour exceeds some contours generated by other scenarios, for example when $\mathrm{B}_{4} \mathrm{C}$ is placed in the beam in the early sections of the detector tank. Therefore these two sections should be handled together when the required shielding thicknesses are determined. 


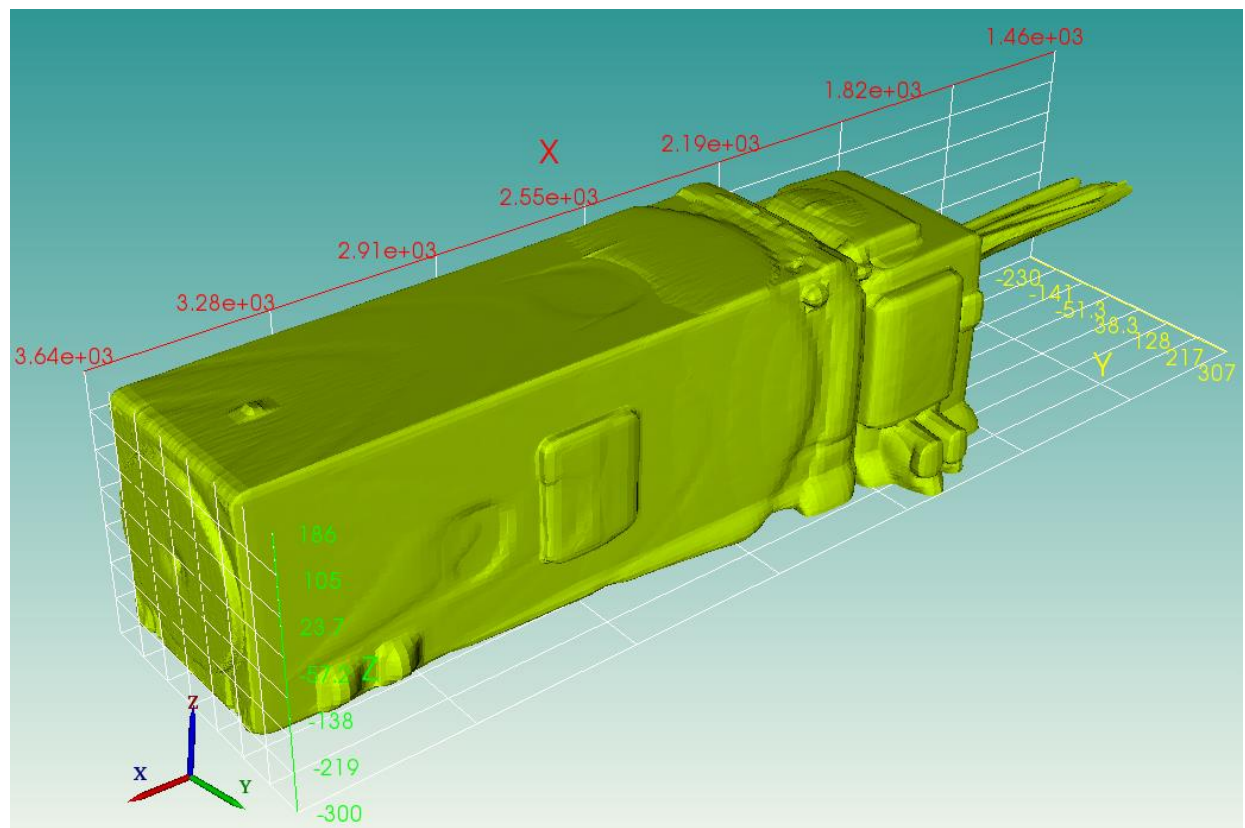

Figure $141.5 \mu \mathrm{Sv} / \mathrm{h}$ gamma dose rate contour surface around the sample and detector tank area. All axes dimension is $\mathrm{cm}$.

\section{Conclusions}

Shielding design of LoKI is a complex process, driven by the radiological requirements while keeping in mind the activation and the instrument background reduction as well. The technical constrains like the available space or floor loading sometimes requires an iterative approach. The combination of available tools like McSTAS, SuperMC and MCNP make the work possible even if the calculation itself is time consuming. Using better spatial resolution of the tallies is paying off in the saving of margins of the shielding and in the determination of hot spots or leaking paths.

\section{References}

[1] R. Garoby et. al., The European Spallation Source Design, Physica Scripta, Volume 93, Number 1. https://doi.org/10.1088/1402-4896/aaecea

[2] A. J. Jackson and K. Kanaki, ESS Construction Proposal LoKI - A broad-band SANS instrument, https://europeanspallationsource.se/sites/default/files/files/document/2017-09/loki_proposal_stc_sept2013.pdf

[3] G. Muhrer and F. Javier, ESS Procedure for designing shielding for safety, ESS-0019931 https://indico.esss.lu.se/event/729/attachments/5694/7902/ESS-0019931_ESS_Procedure_for_designing_shielding_safety.pdf

[4] G. Muhrer and F. Javier, Definition of Supervised and Controlled Radiation Areas, ESS-0001786 https://indico.esss.lu.se/event/740/attachments/5726/8016/Definition-Supervised-Controlled-RadAreas-ESS-0001786.pdf

[5] A. J. Jackson, H1 and H2 design scenarios for the LoKI Instrument, ESS-0114072

[6] H. Wacklin and A. Vickery, ESS Instrument Construction Proposal FREIA, https://ess-publiclegacy.esss.se/sites/default/files/freia_proposal.pdf

[7] C.J. Werner (editor), MCNP Users Manual - Code Version 6.2, LA-UR-17-29981 https://laws.lanl.gov/vhosts/mcnp.lanl.gov/pdf_files/la-ur-17-29981.pdf

[8] Y. Wu, J. Song, H. Zheng, et al. CAD-Based Monte Carlo Program for Integrated Simulation of Nuclear System SuperMC, Annals of Nuclear Energy 82(2015) 161-168 https://doi.org/10.1016/j.anucene.2014.08.058

[9] Y. Wu, FDS Team. CAD-based interface programs for fusion neutron transport simulation, Fusion Engineering and Design 84 (2009) 1987-1992 https://doi.org/10.1016/j.fusengdes.2008.12.041 
[10] K. Lefmann and K. Nielsen, McStas, a General Software Package for Neutron Ray-tracing Simulations, Neutron News 10 , 20, (1999)

[11] P. Willendrup, E. Farhi and K. Lefmann, McStas 1.7 a new version of the flexible Monte Carlo neutron scattering package Physica B, 350 (2004) E735.

[12] P. Willendrup, E. Farhi, E. Knudsen, U. Filges and K. Lefmann, McStas: Past, present and future, Journal of Neutron Research, 17,( 2014), no. 1, pp. 35-43 DOI: 10.3233/JNR-130004

[13] P. Willendrup, and K. Lefmann, McStas (i): Introduction, use, and basic principles for ray-tracing simulations, Journal of Neutron Research, preprint, pp. 1-16, 2019 DOI: 10.3233/JNR-190108

[14] V. Santoro, D. Di Julio, P. Bentley and L. Zanini, Source term for shielding design of bunker and beamlines at ESS, ESS0416080, March 11, 2019

11 [15] R. Kolevatov, C. Schanzer and P. Boeni, Neutron absorption in supermirror coatings: Effects on shielding, Nuclear 12 Instruments and Methods in Physics Research Section A: Accelerators, Spectrometers, Detectors and Associated Equipment, 13 922, 1 April 2019, Pages 98-107 https://doi.org/10.1016/j.nima.2018.12.069 